
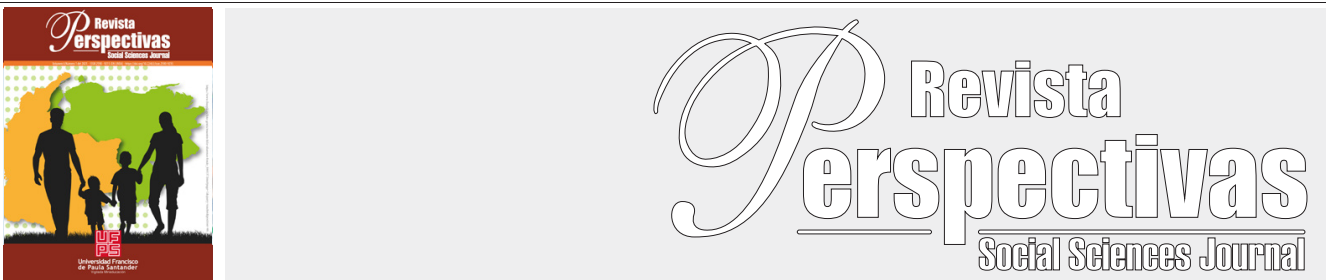

Artículo Original

https://doi.org/10.22463/25909215.2832

\title{
La formación de profesionales de la educación y el cambio en las comunidades rurales a partir de la IAP
}

\section{The training of education and change professionals in rural communities from the IAP}

\section{Celia Carrera-Hernández ${ }^{1}$, Josefina Madrigal-Luna ${ }^{2}$,Yolanda Isaura Lara-García ${ }^{3}$}

${ }^{\prime}$ Doctorado en educación, ccarrera@upnech.edu.mx,ORCID: 0000-0002-2444-2204,Universidad Pedagógica Nacional del Estado de Chihuahua,Ciudad Chihuahua, Chihuahua, México

${ }^{2}$ Universidad Pedagógica Nacional del Estado de Chihuahua Avenida Mirador 4700. Residencial Campestre C.P. 31213 Ciudad Chihuahua, Chihuahua, México, Teléfono 6144107676, Jmadrigal@upnech.edu.mx

${ }^{3}$ Universidad Pedagógica Nacional del Estado de Chihuahua Avenida Mirador 4700. Residencial Campestre C.P. 31213 Ciudad Chihuahua, Chihuahua, México, Teléfono 6144107676, ylara@upnech.edu.mx

Cómo citar: C. Carrera-Hernández, J. Madrigal-Luna, Y.I Lara-García, "La formación de profesionales de la educación y el cambio en las comunidades rurales a partir de la IAP.”. Revistas Perspectivas, 5, No. 2, 72-84, 2020.

Recibido: August 02, 2019; Aprobado: November 15, 2019.

\begin{tabular}{|c|c|}
\hline & RESUMEN \\
\hline $\begin{array}{l}\text { Palabras clave: } \\
\text { Intervención comunitaria, } \\
\text { interventor educativo, } \\
\text { IAP, } \\
\text { cambio social, } \\
\text { formación. }\end{array}$ & $\begin{array}{l}\text { Las comunidades rurales viven problemáticas derivadas de una política económica antidemocrática que pone } \\
\text { en desventaja a los grupos sociales más desfavorecidos, por ello, las instituciones de educación superior } \\
\text { deben desarrollar en sus estudiantes herramientas que se dirijan al logro de cambios sociales orientados a la } \\
\text { búsqueda de calidad de vida y bienestar de la población a partir de la Investigación Acción Participativa (IAP). } \\
\text { El propósito de la investigación es analizar las problemáticas de las comunidades rurales y buscar soluciones a } \\
\text { partir del desarrollo de la IAP en la práctica diaria de formación de los interventores educativos. Participaron } 24 \\
\text { estudiantes, } 15 \text { líderes de la comunidad y } 50 \text { familias. Se fundamentó en la teoría del desarrollo comunitario. Se } \\
\text { utilizó la encuesta, el taller investigativo y la entrevista, se identificaron necesidades y problemas organizados en } \\
\text { grupos etáreos, se propuso un plan de acción que se implementará con el apoyo de instancias gubernamentales } \\
\text { y se reflexionó sobre la influencia de la IAP en la formación como interventores educativos. Se encontró que } \\
\text { la formación teórico metodológica de los universitarios para intervenir en comunidades rurales favorece el } \\
\text { desarrollo de la conciencia social crítica de los estudiantes y la praxis de la investigación a partir de la reflexión } \\
\text { pedagógica de los profesores, situación que impacta en la transformación e innovación curricular. Se concluye } \\
\text { que la formación de profesionales de la educación orientada al cambio social demanda un proceso reflexivo } \\
\text { pedagógico desde la praxis y la transformación social e individual así como el cambio en el currículo formal. }\end{array}$ \\
\hline
\end{tabular}

\section{ABSTRACT}

Keywords:

Community intervention, educational controller, IAP, social change, training.
Rural communities experience problems derived from an undemocratic economic policy that puts the most disadvantaged social groups at a disadvantage, therefore, higher education institutions must develop in their students tools that are aimed at achieving social changes aimed at the search for quality of life and well-being of the population from Participatory Action Research (PAR). The purpose of the research is to analyze the problems of rural communities and seek solutions from the development of PAR in the daily training practice of educational interveners. 24 students, 15 community leaders and 50 families participated. It was based on the theory of community development. The survey, the research workshop and the interview were used, needs and problems organized in age groups were identified, an action plan was proposed that will be implemented with the support of government agencies and reflected on the influence of PAR on training as educational auditors. It was found that the theoretical-methodological training of university students to intervene in rural communities favors the development of critical social awareness of students and the praxis of research from the pedagogical reflection of teachers, a situation that impacts on transformation and innovation curricular. It is concluded that the training of education professionals oriented to social change demands a pedagogical reflective process from praxis and social and individual transformation as well as the change in the formal curriculum. 


\section{Introducción}

La licenciatura en Intervención Educativa diseñada por la Universidad Pedagógica Nacional incluye dentro de sus cursos en la línea profesionalizante de Educación Inclusiva, la intervención en la comunidad, cuya competencia es que los alumnos diseñen, implementen y evalúen un proyecto de intervención comunitaria. Este curso, desde el currículo formal, posibilita a la universidad mantener nexos con la sociedad y promover el desarrollo del pensamiento crítico en estudiantes a partir del análisis reflexivo de la realidad social. De acuerdo con Guevara (2017) las instituciones de educación superior deben desarrollar en sus estudiantes herramientas que se dirijan al logro de cambios sociales orientados a la búsqueda de calidad de vida y bienestar de la población. Debe tener espacios de formación formal y no formal que promuevan la actitud de servicio, hacia la transformación del ser, capaz de transformar y ser transformado. Tema que debe ser pensado desde las universidades por su relevancia social y profesional al igual que la formación disciplinar ya que propicia la vinculación de la universidad con la sociedad con una mirada participativa y crítica hacia el cambio y la transformación. A pesar de ello, la forma tradicional de formación universitaria en la que se pone en práctica el currículo no favorece la vinculación de la universidad con la sociedad ya que la intervención que realizan los alumnos es con un enfoque práctico en el que solo recuperan información y diseñan acciones para solucionar los problemas sin incidir en la participación comunitaria.

Se considera que los alumnos no deben estar ajenos a la realidad social en la que se vive desigualdad en sus diferentes manifestaciones en el contexto mundial, nacional y local, lo cual demanda propuestas educativas, comunitarias y sociales que contribuyan a reducir las brechas existentes entre los diferentes grupos sociales, las cuales impiden que una importante parte de la población tenga acceso a las condiciones básicas para vivir plenamente.
Actualmente, se identifican con facilidad altos niveles de exclusión social hacia las personas de los grupos más desfavorecidos a pesar de los esfuerzos por disminuir la discriminación y las diferencias. Cada vez más aumenta el número de personas que padece pobreza extrema, desnutrición, rezago educativo y discriminación social. Los grupos más desfavorecidos, están destinados a padecer este tipo de problemas, lo cual indica que si se siguen ignorando, será cada vez más difícil el cambio social. Por ello, se hace necesario que los interventores educativos, los profesores de diferentes niveles educativos, investigadores, autoridades políticas y representantes de instancias gubernamentales con orientación social promuevan el desarrollo en las comunidades rurales a partir de la participación, el diálogo y la investigación.

Sin embargo, a pesar de que la investigación acción participativa (IAP) ha sido utilizada en la intervención de numerosas comunidades no se han realizado estudios que aporten al conocimiento de la formación de profesionales educativos para la intervención comunitaria desde la Investigación acción participativa. Por ello, se plantea lo siguiente: ¿Cómo transformar la vida en las comunidades rurales desde la participación de las universidades y de qué manera influye en el cambio curricular y la formación de los interventores educativos?

El objetivo general es influir en la transformación de la vida en las comunidades rurales desde la participación de las universidades y analizar su influencia en el cambio curricular y la formación como interventores educativos.

La perspectiva teórica desde la cual se revisaron los datos es el desarrollo humano desde la intervención socio-comunitaria ya que es una propuesta teórica latinoamericana que se interesa por los problemas sociales y el cambio social, algunos de los modelos teóricos en que se sustenta son el modelo ecológico, el modelo de cambio social y el modelo de empoderamiento (empowerment). 
El modelo ecológico proviene del término ecología desde la mitad del siglo XIX propuesto por Haeckel para hacer mención a la ciencia que estudia la relación de los seres vivos con el medio, sustento reconocido en las ciencias sociales difundido por la Psicología Comunitaria. Lo trascendental de este marco teórico en la intervención comunitaria es la comprensión de interrelación que se establece entre el individuo y los sistemas ambientales en que éste se desenvuelve, la interrelación de estos sistemas constituye un proceso de ajuste, adaptación mutua y acomodación.

Bonfenbrenner (1979/1987 citado en Buelga et al, 2009) define el ambiente ecológico como un conjunto de estructuras concéntricas llamadas micro, meso, exo y macrosistema. El microsistema es el entorno inmediato del individuo conformado por un patrón de actividades, roles y relaciones interpersonales, este entorno tiene características físicas y materiales particulares. El mesosistema es el entorno de relaciones laborales para el adulto, la familia y la vida social mientras que para el niño son el hogar, la escuela y el grupo de amigos. Ente entrono comprende la interrelación de dos o más entornos en los que participa una persona de forma activa. El exosistema es un entorno en el que la persona no participa de forma activa pero influyen en su desarrollo, un ejemplo de ello es el entorno laboral del padre que influye en el desarrollo del niño para su crecimiento o empobrecimiento. El principio fundamental de este enfoque es la colaboración e interrelación entre los diferentes sistemas. La relación de colaboración entre el profesional y los participantes de la comunidad para crear acciones compartidas que ayudan a descubrir y comprender los problemas de la comunidad, por lo que se crea un programa de colaboración.

El modelo de cambio social está ligado a la Piscología social comunitaria en Latinoamérica que nace en los años setenta a partir de una corriente crítica que busca a partir de la reflexión del entorno social la transformación de las comunidades a partir de proyectos societarios comprometidos con comunidades oprimidas y marginadas por lo que se identifica un fuerte compromiso con la justicia social. Los constructos teóricos que subyacen este modelo son derivados de la teoría marxista, del interaccionismo simbólico, de la educación popular y del construccionismo social. Las variables de este modelo son la base material, la ideología, la comunicación, la conciencia, el control y la construcción de la realidad. (Serrano-García et al, 1987 citado por Buelga, 2009).

El desarrollo de la comunidad entonces, representa en el modelo de cambio social un fin y un medio para lograr cambios profundos en la estructura del sistema social a partir de proyectos societarios que respondan a las necesidades y solucionen problemas que de manera consiente identifica la población a partir de un sistema de colaboración y comunicación constante. La intervención consiste en potenciar los recursos con los que cuentan los propios pobladores de las comunidades para enfrentar su identidad como marginados (cultura popular) con capacidad para comprometerse en diferentes ámbitos (personal, grupal y comunitario) para la autoeducación, formación y estrategias de supervivencia.

Es el interventor educativo un profesional de la educación quien con sus conocimientos sobre la intervención comunitaria y el reconocimiento del impacto de las problemáticas sociales, económicas y políticas en las comunidades moviliza sus propios recursos para provocar la reflexión colectiva, la dinamización y cambio de conciencia el auto reconocimiento cultural y social y la búsqueda de acciones para lograr el cambio social.

El empoderamiento también conocido como potenciación o fortalecimiento se define como el proceso porelcual las personas, gruposy comunidades tienen el control y el dominio de sus propias vidas a través de procesos democráticos fortalecidos para el logro de los derechos humanos y la influencia social real. Por ello es importante que los pobladores 
actúen con autodeterminación individual en un ambiente de participación democrática en la vida de la comunidad. Como menciona Hombrados (1996) citado en Buelga et al (2009) el empoderamiento se funda en la idea del poder que tenemos las personas para dirigir nuestras propias vidas y de la capacidad para involucrarse en la vida de la comunidad, lo cual se constituye en el objetivo de los profesionales de la educación, de los psicólogos y demás profesionistas que busquen el cambio en las comunidades. El empoderamiento lleva al logro de la identidad social del individuo no solo en las comunidades sino en la sociedad en general.

\section{La formación universitaria y su vinculación con la comunidad: una relación sistémica}

La comunidad debe mantener una relación muy estrecha con la escuela, sobre todo en los niveles de medio superior y superior, para esta vinculación el currículum formal debe establecer cursos y actividades además de las prácticas profesionales y servicio social que vinculen las actividades de enseñanza y aprendizaje dentro de la comunidad como parte de su formación. Desde esta consideración Ramírez (2017) menciona que la escuela no es un agente independiente del resto de organizaciones sociales que de manera formal tanto implícita como explícitamente incide en la formación de personas de diferentes edades.

Desde el enfoque sistémico se busca articular las actividades comunitarias con el apoyo de proyectos socioeducativos y psicopedagógicos que diseñan estudiantes y profesores dentro de las universidades en comunicación directa con personas de la comunidad y con sus líderes.

Se propone una acción educativa recursiva con experiencias comunitarias que tienen como base la comunicación entre estudiantes y personas de la comunidad para atender necesidades y problemas sentidos en diferentes ámbitos como el pedagógico y el social. El diseño de proyectos podrá ser dirigido a la solución de problemas de diferentes grupos sociales caracterizados por su vulnerabilidad en cuanto a los aspectos de salud, deporte, identidad social de jóvenes, niños descuidados por sus padres, personas desempleadas, jornaleros migrantes, mujeres violentadas, personas con discapacidad, adultos mayores, personas en adicciones $\mathrm{y}$ prostitución, rezago académico, etc. Dicha vinculación sistémica no es fácil de lograr, es una relación compleja, por lo que según Ramírez (2017), las características de los proyectos enfocados desde la visión comunitaria deben concretarse mediante el trabajo colaborativo dentro y fuera del centro escolar $\mathrm{y}$ en el establecimiento de lazos de cooperación y entendimiento entre los diferentes sistemas. Además formar parte del currículum formal y ponerse en práctica a partir de un proceso reflexivo de los profesores para su mejora.

\section{Materiales y métodos}

La presente investigación se realizó desde un paradigma sociocrítico ya que el conocimiento se genera a partir de acciones orientadas a la transformación de la realidad social con una actitud reflexiva y participante tanto del investigador como del grupo que se estudia. Se utilizó la metodología de la IAP, en este artículo solo se da cuenta de las dos primeras fases.

Además se siguió de manera puntual la metodología de intervención comunitaria que propone Mori (2008), constituido por 8 fases a partir de la experiencia de intervención comunitaria durante algunos años. Esta metodología tiene estrecha vinculación con las fases de la IAP de Colmenares (2012) quien menciona que la fase 1 se refiere al descubrimiento de la preocupación temática, la fase 2 a la coconstrucción del plan de acción, la fase 3 corresponde a la ejecución del plan y la fase 4 es la reflexión de la investigación.

La metodología de Mori (2008) incluye más fases ya que presenta de forma detallada las acciones que se desarrollan en el proceso de la investigaciónacción participativa. 


\section{El desarrollo de la investigación acción partici- pativa}

Enla fase 1, llamada diagnóstico dela comunidad, se realizó un diagnóstico en la comunidad a partir de la aplicación de diferentes técnicas como la encuesta en la que se diseñó y aplicó un cuestionario mixto de 8 variables a 50 familias de la comunidad de un sector considerado con mayor vulnerabilidad social y económica. Se utilizó la técnica de la encuesta se interroga a los sujetos con finalidad de obtener de manera sistemática medidas sobre los conceptos que se derivan de una problemática de investigación previamente construida. Se desarrolló un taller investigativo con 15 líderes de la comunidad que trabajan en diversas instituciones El taller tuvo como objetivo sensibilizar a los asistentes sobre la necesidad de su participación de forma colectiva en el desarrollo comunitario, una vez manifestado el interés por colaborar, se identificaron las necesidades y problemáticas así como las propuestas de solución. Además se realizó el análisis de relatos históricos de la comunidad, información que proporcionó el INEGI e información en la web.

En la fase 2 se realizó la revisión del estado del arte para proponer un plan de acción, se identificaron propuestas de solución por grupos etáreos en el contexto internacional y nacional, se analizó la información recuperada, además se tomaron en cuenta las propuestas de los pobladores de la comunidad y finalmente el plan de acción se conformó de 24 proyectos que se implementarán con el apoyo de la Secretaría de Desarrollo Social del Estado ya que apoyarán con recursos para que se cristalice el plan propuesto. Al finalizar esta etapa se reflexionó sobre la experiencia de trabajar la IAP en las comunidades durante la formación como interventores educativos. La fase 3 de implementación del plan de acción y la 4 de reflexión sobre la puesta en práctica y la propuesta de mejora aún no se desarrollan y se presentarán sus resultados posteriormente. Por lo que los resultados de la fase 1 y 2 se presentan enseguida.

\section{Resultados y Discusión}

Una vez analizados los datos se identificaron como problemas centrales los siguientes:

1. Falta de pertenencia social en los jóvenes.

2. Desatención de niños por padres de familia.

3. Abandono de adultos mayores.

4. Exclusión social de jornaleros migrantes.

5. Falta de oportunidad laboral para personas con discapacidad.

\section{Falta de pertenencia de los jóvenes}

Según el Consejo Nacional de Evaluación de la Política Nacional (2018) 1 de cada 9 mexicanos vive en pobreza extrema por lo que esto repercute indiscutiblemente para que se agraven problemas sociales y educativos, además de que en las comunidades rurales y semiurbanas no hay instituciones educativas de nivel técnico y superior para que los jóvenes accedan a una educación técnica o profesional y contribuyan en cambios importantes dentro de la comunidad. Los salarios de sus padres y las pocas oportunidades educativas, laborales, deportivas y artísticas que enfrentan los jóvenes de las comunidades rurales los obliga a realizar actividades ilícitas, delictivas y adictivas e incluso a perder su identidad en la comunidad y sentirse ajenos a ella. La teoría de la identidad social considera que los grupos a los que se pertenece, define a la persona, ya que forman parte de una autovaloración, debido a que va aportando bases para la autoestima, seguridad y determinación que define a la persona (Scandroglio, López y San José, 2008).

Actualmente la comunidad estudiada cuenta con 2081 jóvenes, entre los 15 y 29 años de edad y el $70 \%$ presenta problemas derivados de la fuerte influencia de un entorno que no posibilita su desarrollo integral y le condena a vivir en conflictos y problemas sociales serios para él y para la comunidad por lo que se hace necesario lograr que los jóvenes se sientan identificados y sean parte de una comunidad donde tienen mucho que aportar, por lo que la pregunta que surge es: ¿Cómo lograr que 
los jóvenes adquieran un sentido de pertenencia en grupos sanos que beneficien su desarrollo personal y social en la comunidad? Con el objetivo de lograr que los jóvenes adquieran sentido de pertenencia en grupos sanos que beneficien su desarrollo personal y social se proponen las siguientes estrategias:

Estrategia 1. Gestionar la apertura de un centro de artes y oficios donde los jóvenes aprendan algún oficio para el autoempleo.

Estrategia 2. Taller de identidad para el auto reconocimiento de sus intereses, debilidades $\mathrm{y}$ fortalezas.

Estrategia 3. Cursos de oficios que ofrece el CECATI con el propósito de que los jóvenes mejoren su calidad de vida en las comunidades.

Estrategia 4. Curso de orientación; "un café, buenos amigos y los problemas se vuelven livianos" cuyo propósito es la asignación de un tutor que pueda escuchar, apoyar y orientar a los jóvenes de la comunidad.

Estrategia 5. Crear la fundación Cárdenas para facilitar el acceso a los jóvenes de la comunidad de Lázaro Cárdenas, a psicólogos o trabajadores sociales que les oriente en su formación personal. Además de que se les dé la información necesaria para tener un desarrollo óptimo y que tengan una vida plena.

Estrategia 6. Solicitar la creación de escuelas de carreras técnicas y licenciaturas como el CONALEP Campus Cárdenas y extensiones de facultades de la Universidad, con el propósito de ofrecer a los jóvenes oportunidades de formación técnica y profesional.

\section{La desatención de niños por padres de familia}

Al analizar los datos se identificó la problemática de la "desatención en los hijos por los padres de familia" dentro de la comunidad, se considera que la comunicación de padres con hijos es muy importante es un soporte emocional para cada integrante, puesto que a través de la interacción con sus hijos, los padres proveen experiencias que pueden influir en el crecimiento y desarrollo del niño positiva y negativamente. Según datos del INEGI (2015) sobre la localidad, se identifica que la población total en niños de 0 a 14 años es de 2850 de los cuales el 10\% sufre desatención por padres de familia. La familia es el primer espacio donde los niños tienen oportunidades de desarrollo a partir del funcionamiento familiar (Suárez y Vélez, 2018).

La falta de atención por parte de los padres en los niños sobre todo durante la educación primaria crea en ellos una desmotivación hacia los estudios, y por consiguiente, su rendimiento es menor que en los niños que tienen el apoyo y atención de sus padres. La falta de atención de los padres influye en los niños y niñas creando un desinterés hacia lo que está en su entorno perjudicando su desarrollo en lo personal, social, físico, cognitivo y emocional. Si bien los niños tienen el derecho de recibir los cuidados de sus padres y madres o familiares, aquellos que ya no tienen familia, que han quedado separados de ella, o cuya familia representa un serio peligro para su salud o desarrollo, tienen derecho a recibir cuidados alternativos. En el Artículo 20 de la Convención sobre los Derechos del Niño se mencionan alternativas como: la colocación en hogares de guarda, la adopción o, de ser necesario, la colocación en instituciones adecuadas.

Otro problema derivado de la desatención de los niños es que en la comunidad se encuentran 338 niños de 3 a 5 años y 46 niños de edad de 6 a 11 años que no asisten a la escuela según datos del INEGI (2015). Estos niños demandan atención de sus padres, requieren sentirse motivados por asistir a la escuela y aprender, ser apoyados en el desarrollo de sus actividades para forjar una actitud positiva hacia la escuela, la vida y la comunidad, sentirse aceptados y queridos además de ser escuchados respecto a sus necesidades.

Entre las causas de la desatención a los niños se encuentran los padres separados, el desinterés por la educación y desarrollo de los hijos, descuido de la salud, falta de comunicación, abandono por tiempo ampliado en el trabajo, falta de recursos económicos, madres solas, falta de tiempo, violencia intrafamiliar, 
la falta de apoyos y centros en las comunidades a donde puedan asistir los niños sin costo ya que sus padres generalmente son jornaleros agrícolas. Las consecuencias de la desatención de los padres a los hijos son la desnutrición, manifestaciones de cariño erróneo ya que intercambian cosas materiales por amor, baja autoestima, violencia social, enfermedades mentales, desnutrición, vandalismo, adicciones, situación de calle, embarazos tempranos, bajo rendimiento académico, bajas expectativas escolares y deserción escolar.

La familia es la primera institución que ejerce influencia en el niño, ya que transmite valores, costumbres y creencias por medio de la convivencia diaria. Asimismo, es la primera institución educativa y socializadora del niño, desde que nace comienza a vivir la influencia formativa del ambiente familiar (Guevara, 1996). Es de gran importancia la atención que los padres deben de brindar a sus hijos, es por ello que se busca lograr los siguientes objetivos. Por lo que la pregunta que surge es: ¿Cómo lograr que los padres de familia ofrezcan un mejor cuidado a sus hijos y estén pendientes de su desarrollo en diferentes áreas como la salud, alimentación, seguridad e higiene, educación, etc. para que tengan una mejor calidad de vida? Se reconocen situaciones de desprotección infantil cuando los niños/as tienen las necesidades básicas descubiertas durante un tiempo amplio que le originan daño en su desarrollo, por ello, se propone que los padres ofrezcan una mejor atención y cuidado a sus hijos y estén pendientes de su desarrollo en las diferentes esferas para que los niños tengan una mejor calidad de vida dentro de la familia. Se propone resolver el problema con los siguientes talleres para padres de familia y niños:

Los talleres que se realizarán son los siguientes:

Estrategia 1. Taller con actividades de rompe hielo para padres e hijo

Estrategia 2. Taller crear estrategias para que los niños puedan realizar sus tareas en compañía de sus padres
Estrategia 3. Taller ayudando a mamá en la cocina

Estrategia 4. Taller para mejorar la comunicación entre padres e hijos

Estrategia 5. Taller de arte para los niños y padres.

Estrategia 6. Actividades extraescolares con la participación de los padres

\section{El abandono de los adultos mayores}

El Sistema Nacional para el Desarrollo Integral de la Familia (SNDIF) dio a conocer que en México hay más de 13 millones de personas adultas mayores y que el $16 \%$ sufre rasgos de abandono y maltrato, cuatro de cada diez personas de 60 años o más viven solas $(41.4 \%)$ son económicamente activas, siete de cada diez (69.4\%) personas de edad que viven solas presentan algún tipo de discapacidad o limitación. (El Sol de México, 2019). En el estado de Chihuahua se estima que hay alrededor de 3 millones $500 \mathrm{mil}$ habitantes de los cuales 326,484 son mayores de 60 años, se considera que para el 2030 el 17\% de la población chihuahuense tendrá más de 60 años. Según el Consejo Nacional de la Población el mayor número de personas de tercera edad son mujeres, por cada 100 hombres existen 111 mujeres y se incrementa el número a mayor edad. (Sandoval, García y Richard, s/f).

En la comunidad estudiada habitan 692 adultos de 60 años a más siendo aproximadamente, lo cual representa el $8.4 \%$ de la población total. Según datos del INEGI (2010), el 13\% de la población adulta mayor viven solos y muchos de ellos no tienen apoyo por parte de sus familias, siendo el único ingreso la ayuda económica que les corresponde por ley con la cual no les es suficiente para cubrir sus necesidades básicas. Al ser una minoría en la población total, los adultos mayores suelen ser abandonados por sus mismos familiares por distintos factores que influyen directamente en el cuidado del adulto mayor como lo puede ser la situación económica de la familia, pues no cuentan con los recursos para solventar las necesidades básicas debido al desempleo que 
existe en la localidad, no tienen algún familiar que sea responsable de su cuidado, o bien no hay en las comunidades rurales instituciones de apoyo al adulto mayor dedicadas a mejorar la calidad de vida de estas personas.

Esta situación que viven los adultos mayores de la comunidad debe ser atendida buscando disminuir o si bien eliminar totalmente esta problemática, ya que podría producir efectos negativos en las personas de la tercera edad como inestabilidad emocional que puede llevar al adulto a caer en depresión y llegar a intentos de suicidio en casos más graves, la búsqueda de empleos informales que no son apropiados para su edad y capacidades con el fin de generar ingresos económicos para poder solventar las necesidades básicas e incluso llegar a la indigencia, es decir, adultos en la calle viviendo solamente de los apoyos económicos que les pueden proporcionar las personas generando posibles problemas de desnutrición al no tener recursos para comprar alimentos sufriendo el rechazo de la sociedad y discriminación por encontrarse en condiciones deplorables, por lo que surge la siguiente pregunta: ¿Cómo mejorar la vida de los adultos mayores para que tengan una vida estable integrándose en la comunidad? Con el objetivo de mejorar la vida de los adultos mayores para que tengan una vida estable e integrada en la comunidad se proponen las siguientes estrategias:

Estrategia 1. Creación del centro de cuidados a largo plazo para el adulto mayor con el propósito de proporcionar un lugar para que los adultos mayores en situación de abandono tengan un techo donde vivir.

Estrategia 2. Creación de un comedor comunitario para proveer un lugar donde se den alimentos para las personas que se encuentran en situación de vulnerabilidad como los adultos mayores.

Estrategia 3. Creación del centro de la tercera edad "Jardín del abuelo" para lograr que el adulto mayor tenga un lugar a donde asistir para realizar actividades de su interés y a su vez conviva con otros miembros de la comunidad.

Estrategia 4. Fundación de un huerto comunitario con el propósito contar con un espacio que se encuentre a cargo del adulto mayor para que se sienta productivo.

Estrategia 5. Talleres de concientización dirigidos a la comunidad sobre temas de importancia en relación al adulto mayor con el propósito de sensibilizar a la comunidad sobre la valiosa contribución que realizan las personas adultas, y a su vez conozcan las consecuencias que producen el abandono y descuido del adulto mayor.

\section{La exclusión social de los jornaleros agrícolas migrantes.}

Un problema muy sentido en la comunidad rural donde la actividad principal es la agricultura es la exclusión que sufren los jornaleros agrícolas en México, los cuales se caracterizan por ser un grupo social altamente vulnerable, esto es debido a las insuficientes oportunidades laborales, por la constante movilidad en la que se encuentran al migrar de un estado a otro; la falta de documentos de identificación oficial, así como, las desigualdades y prejuicios que sufren dentro de la comunidad por la pobreza en la que viven, llevándolos a una falta de pertenencia ya que no logran sentirse parte de la comunidad en la que se encuentran. Situación que viven los jornaleros migrantes de la comunidad estudiada, lo cual se caracteriza por un carácter de exclusión social. Las personas jornaleras agrícolas son aquellos trabajadores eventuales del campo que se emplean, a cambio de un salario, en labores que van desde la preparación del terreno, hasta el cuidado y cosecha de cultivos. La migración rural y el mercado agrícola es la única oportunidad de acceder a un empleo y adquirir ingresos para el sostén familiar. (Rojas, 2017).

La exclusión social se refiere a "una serie de procesos en lo que algunas personas y/o grupos 
sociales que se ven apartados de un conjunto de derechos políticos, laborales, económicos y sociales" (Molero, Navas y Morales, 2001, p. 14). Los migrantes se encuentran con barreras sociales pues no se les permite participar en los diversos ámbitos sociales, por lo que viven en desventaja, se les aparta del resto de la comunidad y viven discriminación y marginación, por lo que son un grupo altamente vulnerable.

Según Diez \& López (2019, p. 46) el concepto de exclusión social está muy relacionado con otras condiciones como lo son los prejuicios, la discriminación, la pobreza, la privación y la desigualdad. De acuerdo a la Encuesta Nacional de Migración de la Universidad Nacional Autónoma de México (UNAM), los migrantes son el grupo al que más desconfianza se le tiene, lo que deriva en actos discriminatorios, como la violación de sus derechos humanos; derecho al trato digno, a la igualdad de oportunidades, derecho al trabajo; robos, secuestros, detenciones injustas, la falta de acceso a servicios básicos (atención medica), malos tratos y salarios bajos. Por este tipo de condiciones se puede considerar que los jornaleros migrantes son un grupo excluido dentro de la sociedad en la que se desenvuelven.

Además, la mayoría de los migrantes no cuentan con documentos oficiales de identificación, tales como el acta de nacimiento, la clave única de registro de población (CURP) ni la credencial del Instituto Nacional Electoral (INE) las cuales son necesarias para poder acceder a los servicios básicos. Prácticamente, los migrantes no existen dentro de la sociedad, sin mencionar que en la comunidad del estudio no se cuenta con un registro del número de migrantes que la habitan actualmente.

En consecuencia, se ven obligados a trabajar sin contratos formales, dejando al trabajador desprotegido, al no gozar de aguinaldo, prestaciones y otros derechos que les brinda la ley, sin mencionar que estos reciben salarios muy bajos, insuficientes para cubrir los gastos; por lo tanto, no logran acceder a los servicios básicos, como lo es la atención médica y el contar con una vivienda, por lo que terminan viviendo en las calles y sufriendo de actos discriminatorios por parte de la comunidad lo que resulta en la violación de sus derechos humanos.

Como mencionan Molero, Navas y Morales (2001) la exclusión social hace referencia a una serie de procesos en virtud de que algunas personas y grupos sociales se ven apartados de un conjunto de derechos de carácter político, laboral, económico y social. Este concepto está muy relacionado con otros estigmas como el prejuicio, discriminación, pobreza, privación y desigualdad de los derechos de las personas. En resumen, está población vive excluida y separada de la nación, por lo que es de gran importancia resolver este problema. La pregunta es ¿Cómo lograr que los jornaleros migrantes sean integrados a la comunidad? Con el objetivo de lograr la inclusión social de los jornaleros migrantes en la comunidad estudiada se propone como plan de acción las siguientes estrategias:

Estrategia 1. Realizar un censo de jornaleros migrantes para contar con un registro y conocer su identidad, saber quiénes son, edad, sexo, lugar de origen, lengua, etc. y poder brindarles a los jornaleros agrícolas pláticas sobre los beneficios que se les pueden ofrecer teniendo un documento de registro.

Estrategia 2. La comunidad y los jornaleros migrantes con el propósito de crear interés entre los habitantes de la comunidad en la organización de un comité ciudadano para que puedan apoyar a los jornaleros agrícolas migrantes, de tal manera que la misma comunidad participe en un grupo de apoyo para las personas migrantes.

Estrategia 3. Apoyo de jóvenes bilingües como traductores para apoyar en el área de la salud y educación debido al dominio del español como segunda lengua.

Estrategia 4. Taller de capacitación para jornaleros migrantes en el albergue de la comunidad 
para la realización de diferentes actividades como cocina, repostería, etc. con el propósito de buscar el arraigo de las personas con el autoempleo, además crear un comedor para personas migrantes de manera colaborativa.

Estrategia 5. Mayor acceso a servicios médicos y asesoramiento legal a jornaleros agrícolas migrantes de la comunidad con el fin de que conozcan sus derechos y soliciten su cumplimiento.

5. La falta de oportunidades laborales para personas con discapacidad

En las comunidades rurales las fuentes de empleo son muy escasas y predomina el trabajo en el campo o en la industria maquiladora en las ciudades cercanas, la albañilería y el comercio en pequeña escala. Además, las personas con discapacidad no son empleadas debido a que no hay infraestructura para facilitar su desplazamiento. La ausencia de oportunidades de empleo, la falta de sensibilización de los habitantes de la comunidad sobre las posibilidades de desarrollo en el ámbito laboral y la falta de infraestructura tienen una gran influencia en la marginación y pobreza de las personas con discapacidad y sus familias.

Sin embargo, dada la importancia de que las personas con discapacidad cuenten con un empleo el Programa Nacional de Trabajo y Empleo para las Personas con Discapacidad (2014- 2018) publicado en el Diario Oficial de la Federación el 28 de abril de 2014, promueve medidas para garantizar la inclusión laboral de este grupo de población en situación de vulnerabilidad, mediante el ejercicio pleno de su derecho social a un trabajo digno.

Reynoso (2006) señala que el trabajo, además de ser una actividad económica, representa un espacio de desarrollo y desenvolvimiento del trabajador, las personas con discapacidad constituyen uno de los grupos más vulnerables con respecto a su integración al mercado laboral, una menor capacidad física o mental está considerada como uno de los principales factores que generan situaciones de desigualdad y que pueden ser causa de discriminación laboral. Es por eso que el trabajo para la personas con discapacidad es un paso transcendente hacia el logro de la autonomía, en el mundo laboral, las personas con discapacidad se encuentran en una lucha constante para obtener las mejores condiciones laborales y superar las barreras que impiden la oportunidad laboral que ellos necesitan, la falta de oportunidad laboral es una violación de sus derechos.

Para solucionar el problema se propone lo siguiente:

Estrategia 1. Creación de un Centro comunitario con el propósito de ofrecer un espacio donde niños, jóvenes, adultos mayores y personas con discapacidad puedan participar en cursos y talleres para propiciar el desarrollo de actividades económicas como oficios con el fin de auto emplearse, además de podrán realizar actividades artísticas y deportivas que contribuyan a su desarrollo integral.

Estrategia 2. Escuela de Artes y oficios para personas con discapacidad con el propósito de que las personas con discapacidad desarrollen habilidades en algún oficio para el autoempleo.

La influencia de la participación comunitaria de los interventores educativos en su formación profesional y en el currículo.

El análisis de los datos que arrojó la entrevista individual y la reflexión grupal favoreció la construcción de la categoría de la conciencia social durante el proceso de formación universitaria como un dispositivo indispensable para el ejercicio de cualquier carrera profesional. La reflexión sobre los problemas identificados, las soluciones propuestas, el diseño de instrumentos, el diálogo con las personas de la comunidad y la reflexión teórica y empírica constante tuvo una fuerte influencia en la formación de los interventores educativos porque 
además de aprender sobre la metodología de la IAP, la teoría de las comunidades y los enfoques de desarrollo comunitario, se aprendió sobre la estructura y organización comunitaria, los problemas sociales económicos y políticos de las comunidades, el desarrollo de actitudes adecuadas para convivir y aprender de la población, escuchar sus ideas y reflexiones sobre la comunidad, analizar propuestas y situaciones, realizar búsquedas de información y aceptar a las personas con sus diferencias, lo cual les llevó a reconocer que como profesionistas hay muchas actividades que desarrollar en las comunidades y que el currículo formal universitario carece de elementos de formación integral pero sobre todo de una orientación para desarrollar la IAP en escuelas, familias y comunidades, entornos donde los profesionistas de las diferentes carreras universitarias pueden incidir con su juventud, energía disposición y conocimiento en la solución de problemas de diferentes grupos etáreos y por ende en el cambio social y en una mejor calidad de vida de estos grupos y el desarrollo de las comunidades en general además como centro la participación y colaboración de todos sus habitantes lo cual hace más dinámica la vida comunitaria en un ambiente democrático.

Los estudiantes reconocen que el trabajar la IAP en la universidad donde se les involucra en el estudio de los problemas y en la búsqueda conjunta de soluciones se genera una fuerza colaborativa importante en los alumnos que surge de un proceso de concienciación que no se logra en las aulas, sino en la interacción y el diálogo con las personas de las comunidades, donde al escucharlos, convivir y participar juntos en la identificación de los problemas contribuye al logro de una conciencia social. "Ahora soy más consciente de lo que viven muchas familias, del compromiso que tengo como interventora y de la necesidad de colaborar con los demás" (E320052020). La conciencia social se forma en la dialéctica de lo concreto de la realidad social en la que se integra la persona, es decir, la transformación de la realidad implica un proceso psicológico en el que se posiciona al individuo en un ejercicio dialéctico de auto reflexión, consigo mismo y en su relación con la realidad. Esta experiencia resulta muy enriquecedora y formativa para el estudiante porque se hacen más sensibles y conocedores de la realidad que viven los diferentes grupos etáreos en las comunidades. "Fue una experiencia muy grata en mi formación en la que realmente me doy cuenta de lo que es un interventor educativo y de la importancia del ámbito comunitario como un espacio muy importante para trabajar por el desarrollo de la población y el cambio en sus vidas" (E823062020).

El paso de la conciencia ingenua a una conciencia crítica es lo que posibilita al individuo a participar de forma constructiva en la transformación de la comunidad. El lograr la conciencia crítica del individuo es terminar con fanatismos y enajenación que paraliza el desarrollo de las comunidades, por ello, el conocimiento intuitivo de la intervención comunitaria se ve superado cuando los alumnos acceden a la reflexión teórico-metodológica de la IAP y la ponen en práctica. El uso de la teoría en la práctica y la teorización de la práctica es un proceso reflexivo de gran importancia para los estudiantes universitarios pues al preguntar sobre las actividades pedagógicas que consideraban más importantes en su formación fue el implementar la IAP en las comunidades e interactuar con las familias, actividad que les dejó aprendizajes más profundos porque además de ser una herramienta para intervenir en las comunidades forma parte de un estilo de vida diferente en el que el saber escuchar, analizar y participar de forma colaborativa transforma no solo a las comunidades sino la vida personal de cada estudiante, no solo como profesional sino como persona. "Ahora veo todo de otra manera, soy diferente, veo lo valioso de la vida y de los demás, pero creo que como grupo somos más humanos" (E114062020). 


\section{Conclusiones}

Con la metodología de la IAP en sus dos primeras etapas, se logró propiciar la participación de la población para iniciar con acciones de impacto en la transformación de su comunidad, además, en la universidad se generó el espacio de discusión sobre la formación de estudiantes conocedores de su realidad, investigadores y profesionales en la propuesta de soluciones para generar el cambio social con pensamiento crítico. Se logró avanzar de una perspectiva técnica del currículo hacia una crítica donde los profesores y los estudiantes son investigadores, reflexivos y participan de forma colaborativa con la comunidad.

Los interventores educativos pudieron conocer de manera directa que las comunidades rurales les demandan su compromiso y participación ya que se caracterizan por la extrema pobreza, el desempleo, la migración constante, la falta de clínicas y servicios de salud, la falta de escuelas de nivel técnico y superior, el rezago educativo, la violencia, la falta de servicios públicos, etc. Problemas que afectan a la población desde muchos años atrás, en los que no se ha atendido el desarrollo social, la organización comunitaria y el desarrollo de programas y proyectos de intervención comunitaria con tendencia al desarrollo y transformación social con la participación de la población. Las acciones de los programas son con fines asistencialistas para atender a propósitos partidistas, por lo cual "las personas no confían actualmente de las diferentes propuestas que puedan recibir para mejorar la vida en las comunidades" (Testimonio habitante de la comunidad), ante ello, involucrarse en la participación para la búsqueda de soluciones con la intención de reconstruir la vida en las comunidades rurales del estado a través de la vinculación de las universidades con alto sentido social, las instancias gubernamentales y las comunidades para que a través de proyectos construidos con la participación de la comunidad tiendan a resolver sus problemáticas y atender sus necesidades sociales ya que actualmente se aprecia una escasa participación en la solución de sus problemas y una amplia dependencia de diferentes instancias para encauzar la solución a sus problemas.

La poca participación de la población ha favorecido el individualismo, el escaso sentido de pertenencia, la ausencia de confianza entre la ciudadanía, la fragmentación comunitaria, la ausencia de una conciencia colectiva para encarar asuntos comunitarios, la escasa conciencia crítica de su realidad e implicación para su transformación, éstos son efectos de una práctica política centrada en la atención a las necesidades de otros grupos sociales y el abandono o el olvido a los grupos desfavorecidos, además, la falta de vinculación entre diferentes instancias, investigadores, docentes y profesionistas dispuestos a implicarse en la participación activa de las comunidades.

Para ello, es indispensable que estudiantes y profesores universitarios desarrollen programas y proyectos comunitarios y que cuenten con una conciencia social, de apoyo mutuo, respeto a las diferencias y conocimiento sobrelas diferentes formas de intervención comunitaria. Que las universidades provoquen una formación profesional de educadores sensibles, con conciencia social a través de una formación centrada en la praxis y que posibilite desde el currículo y las prácticas pedagógicas la IAP para el logro de un perfil del egresado con potencial para propiciar la transformación y el cambio social no solo en comunidades rurales sino en semiurbanas y urbanas. Por ello, se abre una nueva línea de investigación sobre la reflexión curricular crítica orientada a la transformación social desde las universidades que parta de la reflexión sobre la prácticas pedagógica en este nivel.

\section{Referencias}

Buelga, S. et al. (2009). Psicología social comunitaria. Colombia: Trillas.

Colmenares, A.M. (2012). Investigación-acciónparticipativa: una metodología integradora del 
conocimiento y la acción. Voces y silencios. Revista Latinoamericana de Educación. Volumen 3. No. 1. Junio https://doi.org/10.18175/ vys3.1.2012.07

Diez, J. \& López, A.M. (2019). Exclusión social de los vecinos en una perspectiva comparada mundial. Revista Española de Investigaciones Sociológicas, 166: 45-64. (http://dx.doi. org/10.5477/cis/reis. 166.45

El Sol de México (2019). El 16\% de adultos mayores en México sufren abandono y maltrato: SNDIF. Miércoles 28 de agosto en sección de sociedad.

Guevara, G. (1996). La relación familia-escuela. Revista Educación (8), 6-13.

Guevara, C. (abril-septiembre, 2017). Servicio comunitario, aprendizaje-servicio y formación social: claves para la construcción de espacios transformativos universitarios. Revista Electrónica de Humanidades, Educación y Comunicación Social, 23, 85-87. Recuperado el 16 de diciembre de 2020 en: http://ojs.urbe.edu/ index.php/redhecs/article/view/714

INEGI (2015). Encuesta intercensal. Recuperado el 7 de febrero en: www.inegi.org.mx

Molero, F., Navas, M. y Morales, F. (2001). Inmigración, prejuicio y exclusión social: reflexiones en torno a algunos datos de la realidad española. Revista Internacional de Psicología y Terapia Psicológica, I (1), 11-32. [Fecha de consulta 21 de junio de 2020]. ISSN: 1577-7057. Disponible en: https://www.redalyc. org/articulo.oa? $\mathrm{id}=560 / 56001102$

Mori, M.P. (2008). Una propuesta metodológica para la intervención comunitaria. LIBERABIT, 14: 81-90, Lima (Perú) recuperado el 06 de diciembre de 2019 Recuperado de: http://www. scielo.org.pe/pdf/liber/v14n14/a10v14n14.pdf
Ramírez, A. A. (2017). La educación con sentido comunitario; reflexiones en torno a la formación del profesorado. Educación Vol. XXVI, N ${ }^{\circ}$ 51, septiembre 2017, pp. 79-94 / ISSN 10199403 Recuperado de: https://doi.org/10.18800/ educacion.201702.004

Rojas, T. J. (2017). Migración rural jornalera en México: la circularidad de la pobreza. Iberoforum Revista de Ciencias Sociales de la Universidad Iberoamericana, XII (23), 1-35. [Fecha de consulta 21 de junio de 2020]. Recuperado de: https://www.redalyc.org/ articulo.oa? id $=2110 / 211053027001$

Sandoval, J.M., García, M.L. y Richard, M.P. (s/f). Problemática de los adultos mayores en relación a pensiones. Sistema integral de Información y comunicación (SIID). México.

Scandroglio, B. y López, J. S. y San José, M. C. (2008). La Teoría de la Identidad Social: una sintesis crítica de sus fundamentos, evidencias y controversias. Psicothema, 20 (1), 80-89. [Fecha de consulta 21 de junio de 2020]. ISSN: 02149915. Recuperado de: https://www.redalyc.org/ articulo.oa? $\mathrm{id}=727 / 72720112$

Suárez, P., Vélez, M. (2018). El papel de la familia en el desarrollo social del niño: una mirada desde la afectividad, la comunicación familiar y estilos de educación parental. Revista Psicoespaocios, 12(20): 173- 198, Recuperado de: https://doi. org/10.25057/issn.2145-2776 